\title{
Immunohistology of ectopic secondary lymph follicles in subcutaneous nodules from patients with hyperreactive onchocerciasis (sowda)
}

\author{
Norbert W. Brattig • Klara Tenner-Racz • \\ Simone Korten • Achim Hoerauf • Dietrich W. Büttner
}

Received: 20 April 2010 /Accepted: 30 April 2010/Published online: 4 June 2010

(C) The Author(s) 2010. This article is published with open access at Springerlink.com

\begin{abstract}
Ectopic secondary lymph follicles emerge in patients with autoimmune or infectious diseases, e.g. in the synovium in rheumatoid arthritis or the skin in Borrelia burgdorferi infection, but ectopic localisations in the skin are rarely described for helminth infections. We investigated the cellular composition of secondary lymph follicles in subcutaneous nodules from eight patients with hyperreactive onchocerciasis (synonymous "localised" form or sowda) using immunohistology. CD3- and CD45RO-positive $\mathrm{T}$ cells and CD20-positive $\mathrm{B}$ cells were present in the mantle zone. The germinal centre was characterised by many B cells
\end{abstract}

Electronic supplementary material The online version of this article (doi:10.1007/s00436-010-1912-0) contains supplementary material, which is available to authorized users.

N. W. Brattig ( $\square)$

Department of Molecular Medicine,

Bernhard Nocht Institute for Tropical Medicine (BNI),

Bernhard-Nocht-Str. 74,

20359 Hamburg, Germany

e-mail: nbrattig@bni-hamburg.de

K. Tenner-Racz

Department of Pathology,

Bernhard Nocht Institute for Tropical Medicine (BNI),

Bernhard-Nocht-Str. 74,

20359 Hamburg, Germany

S. Korten · D. W. Büttner

Department of Helminthology,

Bernhard Nocht Institute for Tropical Medicine (BNI),

Bernhard-Nocht-Str. 74,

20359 Hamburg, Germany

\section{A. Hoerauf}

Institute of Medical Microbiology,

Immunology and Parasitology (IMMIP), University Clinic Bonn,

Sigmund-Freud-Str. 25,

53105 Bonn, Germany and CD35-positive follicular dendritic cells, which formed a network of attached IgE- and CD23-positive cells with the low-affinity IgE (epsilon) receptor. Few of the B cells were labelled for IgG1, IgG2 and IgG4, whereas in other zones of the nodule IgG1 was expressed by plasma cells and IgG1coated dead microfilariae. B cells and few macrophages expressed the MHC class II molecule HLA-DR. Mature CD68-positive tingible body macrophages with phagocytosed leukocytes and CD57-positive lymphocytes occurred in the germinal centre. Macrophages in the germinal centre only weakly expressed alpha1-antichymotrypsin in contrast to macrophages in other zones of the onchocercoma. Furthermore, the multifunctional cytokine TGF-beta was only weakly expressed by macrophages and lymphocytes in the secondary follicles. Only few tryptase-positive mast cells, calprotectinpositive young macrophages, eosinophils and neutrophils occurred in the secondary follicles, although these cells were abundant in the onchocercomas. In conclusion, the ectopic secondary lymph follicles in onchocercomas and lymph nodes from hyperreactive onchocerciasis patients are equally composed.

$\begin{array}{ll}\text { Abbreviations } \\ \text { ESM } & \text { Electronic supplementary material } \\ \text { FDC } & \text { Follicular dendritic cells } \\ \text { mf } & \text { Microfilaria (-e) } \\ \text { SLF } & \text { Secondary lymph follicle (s) }\end{array}$

\section{Introduction}

Mainly occurring in tropical Africa, chronic infection with the filarial nematode Onchocerca volvulus producing microfilariae (mf), which cause diseases of skin, lymph 
nodes and eyes (World Health Organization, 1987). Individuals only harbouring non-productive female worms do not show signs of disease apart from the onchocercoma, which contains very few immune cells (Specht et al. 2009; Wildenburg et al. 1996). The disease depends on the presence of $\mathrm{mf}$ or dead adult worms and the severity varies with the mf load, the immune status of the patients, the geographic region and on co-infections, but probably not or not much on the strain of $O$. volvulus. The observation of the disease variation led to the notion of a spectrum of clinical and immunological forms (Brattig 2004; Hoerauf and Brattig 2002; Connor et al. 1983; World Health Organization, 1987). The most frequently observed polar form of the spectrum represents the immunosuppressed hyporeactive patients (synonymous generalised form) with high counts of adult worms and $\mathrm{mf}$, which are not eliminated in untreated patients, whereas these individuals are able to eliminate up to $90 \%$ of the infective larvae. The other pole is represented by the only rarely observed hyperreactive patients (synonymous localised form, hyperergic form or sowda(h)) with very low loads of adult worms and $\mathrm{mf}$, because the host eliminates the $\mathrm{mf}$ by generation of local and humoral immune responses. These patients are characterised by a strong local and systemic Th2-type biased immune reaction (Brattig 2004; Hoerauf and Brattig 2002) often causing severe disease. The large group of patients with intermediate forms between the two poles (Rubio de Krömer et al. 1995) comprises recently infected children and immigrants into endemic areas and other not hyperreactive patients, living in hypoendemic areas with low loads of adult worms and $\mathrm{mf}$. These patients are still able to kill the mf without treatment indicated by their repeated attacks of acute papular dermatitis showing damaged $\mathrm{mf}$ in the skin. They may be termed "reactive" in contrast to hypo- or hyperreactive. Patients with chronic infections may experience different forms during their life. Studying onchocercomas by immunohistology (e.g. Albiez et al. 1985; Büttner et al. 1988; Korten et al. 1998), we observed a small group of hyperreactive patients who had developed ectopic secondary lymph follicles (SLF) in their onchocercomas with production of $\mathrm{mf}$, which were killed in these nodules and the skin. The onchocercomas from hyperreactive patients with only one or two pairs of mf-producing worms present a much larger infiltration of immune cells than those with many worms from hyporeactive patients (Büttner and Racz 1983).

SLF occur in all lymphatic organs as lymph nodes and spleen and they are frequently elicited by infections in the intestinal tract from the salivary glands to the large intestine, e.g. in Helicobacter pylori infections of the stomach (Mazzucchelli et al. 1999). Ectopic SLF develop in other organs than the lymphatic system in autoimmune and infectious diseases. Examples for autoimmune conditions are the muscles in juvenile dermatomyositis (López de Padilla et al. 2009) and rheumatoid synovitis (Shi et al. 2001; Timmer et al. 2007). An example for a bacterial infection is the SLF, which was observed in all cutaneous lesions excised from 106 patients with a proven Borrelia burgdorferi infection (Colli et al. 2004). Occasionally, ectopic SLF are seen after application of viral and bacterial vaccines (Cerroni et al. 2007; Maubec et al. 2005). However, there are only rare reports of ectopic SLF in the subcutis and cutis due to helminth infections of human patients. Ratnatunga and de Witesundera (1999) briefly mention SLF observed in subcutaneous nodules from human patients with Dirofilaria repens. Here we describe the types of immune cells observed in ectopic SLF in onchocercomas from human patients with hyperreactive onchocerciasis and also several inflammation and immunoregulation markers such as TGFbeta, HLA-DR, alpha1-antichymotrypsin, histamine, IgE and IgG subclasses and compare the findings with those in nodules from hyporeactive patients (Korten et al. 2010b) and lymph nodes.

\section{Materials and methods}

\section{Patients}

Previously described criteria were used in this study for the diagnosis of hyperreactivity (Albiez et al. 1985; Büttner et al. 1982; Korten et al. 2010a). Briefly, patients with a medical history lasting longer than a year complained of strong pruritus, presented with a chronic papular and often lichenified onchodermatitis, an onchocercoma confirmed by excision, large regional lymph nodes, a positive local Mazzotti test with fresh papules (Darge and Büttner 1995), no or less than $10 \mathrm{mf}$ in the skin snip and usually more than $10 \%$ eosinophils in the blood.

Patients with the hyperreactive form of onchocerciasis often suffer from severe skin disease. Therefore, these patients were offered courses of low diethylcarbamazine doses at the beginning of the study and later ivermectin treatment in the Liberia Research Unit of the BNI from 1982 to 1990 (Albiez et al. 1985; Darge and Büttner 1995; Erttmann, 1987). Nodule carriers were also offered nodulectomies (Albiez et al. 1988), which often improve or cure the symptoms of hyperreactive patients until new worms are acquired and become productive. Sixty-three onchocercomas from 49 hyperreactive patients were extirpated and examined using histology. Among these, SLF were found in seven nodules from seven female patients. The onchocercomas were used for this study together with one nodule from a German boy, who had acquired the infection in Cameroon (Table 1). The patients had presented a moderate to severe disease at the time of nodulectomy or at least 
Table 1 Characteristics of Liberian patients and one German boy with hyperreactive onchocerciasis whose onchocercomas contained ectopic secondary lymph follicles

\begin{tabular}{|c|c|c|c|c|c|c|c|c|c|}
\hline Patient no. & $\begin{array}{l}\text { Sex, age } \\
\text { (years) }\end{array}$ & $\begin{array}{l}\text { Duration } \\
\text { of disease } \\
\text { (years) }\end{array}$ & $\begin{array}{l}\text { No. of } \\
\text { lymph } \\
\text { nodes } \\
\text { swollen }\end{array}$ & $\begin{array}{l}\text { No. of } \\
\text { nodules, site }\end{array}$ & $\begin{array}{l}\mathrm{Mf} / \mathrm{mg} \\
\text { skin }\end{array}$ & $\begin{array}{l}\text { Topical Mazzotti } \\
\text { reaction (degree) }\end{array}$ & $\begin{array}{l}\text { Eosinophils } \\
\text { (number/ } \mu \mathrm{l} \text {; } \\
\text { and \%) }\end{array}$ & $\begin{array}{l}\text { Disease } \\
\text { severity }\end{array}$ & $\begin{array}{l}\text { Treatment } \\
\text { before } \\
\text { nodulectomy }\end{array}$ \\
\hline 1 & $\mathrm{~F}, 7$ & 2 & 4 & 1 , iliac crest & 0 & +++ & 1,$597 ; 22 \%$ & Moderate & DEC \\
\hline 2 & $\mathrm{~F}, 10$ & 1 & 1 & 1 , iliac crest & 0.2 & ++ & 2,$240 ; 26 \%$ & Moderate & DEC \\
\hline 3 & F, 13 & 2 & 2 & 3 , trochanter & 4.6 & + & 2,$278 ; 25 \%$ & Moderate & None \\
\hline 4 & F, 17 & 3 & 0 & 3 , trochanter & 0 & + & 2,$522 ; 26 \%$ & Severe & None \\
\hline 5 & F, 18 & 6 & 3 & 3 , iliac crest & 0.5 & +++ & $825 ; 21 \%$ & Moderate & None \\
\hline 6 & F, 22 & 11 & 3 & 1 , iliac crest & 0.9 & +++ & n. d. & Severe & None \\
\hline 7 & $\mathrm{~F}, 27$ & 2 & 0 & 1 , trochanter & 0 & + & $810 ; 12 \%$ & Mild & DEC \\
\hline 8 & M, 9 & 2 & 3 & 1 , iliac crest & 0 & +++ & n. d. & Moderate & Ivermectin \\
\hline
\end{tabular}

$F$ female, $M$ male, $D E C$ diethylcarbamazine, $n . d$. not determined

before the diethylcarbamazine treatment (for classification see Büttner et al. 1982). The nodules with SLF were compared with onchocercomas and lymph nodes from other hyperreactive and also from hyporeactive and intermediate patients living in Liberia, Yemen and other countries.

\section{Immunohistochemistry}

Onchocercomas had been fixed in $4 \%$ buffered formaldehyde solution and the nodule of a German boy in $80 \%$ ethanol, embedded in paraffin and stained with haematoxylin and eosin, Giemsa and Movat stains. Dewaxed sections underwent antigen retrieval according to the advices of the manufacturers providing the primary antibody. For CD3, CD20 and CD57 staining the sections were pretreated by boiling in citrate solution at $\mathrm{pH} 6$ and for CD35 in EDTA solution at $\mathrm{pH} 8$. Monoclonal antibodies (mabs) were used to characterise most of the immune cells: CD68 for mature macrophages (clone PG-M1, 1:50), calprotectin for young macrophages (L1-protein, MRP8/MRP14, clone MAC387, 1:50), mast cell tryptase (clone AA1, 1:200), CD3 for all $\mathrm{T}$ cells (clone UCHT1, 1:400), CD20 for B cells (clone L26, 1:50), CD23 for the low-affinity IgE (epsilon) receptor FceR-II, clone MHM6, 1:30), CD45RO for a subset of activated $\mathrm{T}$ cells (clone UCHL1, 1:50, all previous antibodies from DakoCytomation, Hamburg, Germany), MHC class II molecule HLA-DR (clone TAL.1B5, 1:30, R\&D Systems, Wiesbaden, Germany), defensin for neutrophils (1:4,000, Dianova, Hamburg, Germany), eosinophil cationic protein for eosinophils (ECP EG2, 1:50, Kabi Pharmacia, Uppsala, Sweden), CD57 for natural killer and some T cells (clone HNK-1, 1:20, Becton Dickinson, USA), CD35 for follicular dendritic cells (FDCs, clone RLB25, 1:60, Novocastra, Newcastle upon Tyne, UK), IgG1 (clone HP-6001, 1:200, Sigma, Deisenhofen, Germany), IgG2 (clone HP-
6002, 1:200, Sigma) and IgG4 (clone HP-6025, 1:1,000, Sigma). We applied polyclonal antibodies (pabs) from rabbits against: Dirofilaria immitis Wolbachia surface protein to demonstrate Wolbachia endobacteria (DiWSP, 1:1000, from Prof. Claudio Bandi, Università degli Studi di Milano, Milano), alpha1-antichymotrypsin (1:500; DakoCytomation), $\operatorname{IgE}(1: 2,000$, DakoCytomation), IgA (1:10,000, Sigma), cathepsin G for neutrophils (1:50, ICN, Biomedicals, Mannheim, Germany) and histamine (1:250, Chemicon, Temecula, USA). For most mabs and the pabs the alkaline phosphataseanti-alkaline phosphatase technique and for histamine the peroxidase-anti-peroxidase complex were applied according to the protocols from DakoCytomation. For CD3 and CD35 amino-9-ethylcarbazole (AEC) kits were used (PolyLink, Peroxydase Label horseradish peroxides (HRP), DCS Innovative Diagnostik Systeme, Hamburg, Germany). Not all sections from the nodules were stained with the differentiation markers mentioned above. For labelling the multifunctional cytokine TGF-beta sections from formaldehyde-fixed nodules were incubated with pabs produced in goats immunised with $\mathrm{CHO}$ cell-derived, recombinant human TGF-beta 1 latency-associated peptide (1:200, R\&D Systems). A pre-diluted biotinylated anti-goat mouse mab served as secondary antibody and for visualisation, incubation with streptavidin-HRP and developing with the brown chromogenic substrate AEC were used (HRP-AEC Cell \& Tissue Staining Kit, R\&D Systems).

\section{Results}

The nodules were onchocercomas

The eight nodules with SLF were all onchocercomas and not lymph nodes. They had been excised from the iliac crest 
or trochanter (Table 1), contained one or two pairs of adult $O$. volvulus, were enclosed by a fibrous pseudocapsule, blood vessels entered the nodule from all sides and not from a hilus, they possessed no trabeculae, no subcapsular and intermediate sinuses and no typical medulla. We never observed adult $O$. volvulus in lymph nodes. All adult worms harboured DiWSP-positive Wolbachia endobacteria.

The onchocercomas were typical for hyperreactive patients

The eight onchocercomas showed the characteristic features of nodules from hyperreactive patients. They were all large and the four of the eight nodules, which were weighed in Liberia, had weights of $1.7,2.8,4.1$ and $4.6 \mathrm{~g}$. In contrast, only $3 \%$ of 1,830 onchocercomas from hyporeactive patients in Liberia weighed more than $1.3 \mathrm{~g}$ (Albiez et al. 1988). In the onchocercomas from hyperreactive patients a small zone with the worms was surrounded by an infiltrate much larger than in hyporeactive nodules (Figs. 1 and 2). In these infiltrates several or many SLF occurred (Figs. 2, 3, 4 and 5) presenting networks of IgE (Fig. 3) and HLA-DR (Fig. 4) in the germinal centre, with $\mathrm{T}$ lymphocytes adjacent to the mantle zone (Fig. 5) and many B cells. Sixty nodules from 36 other hyperreactive patients extirpated in Liberia and 14 nodules from five patients in Yemen showed multiple small infiltrations of lymphocytes but no SLF. Furthermore, we did not find any SLF in more than 1,000 nodules from hyporeactive patients, which we examined during several studies after we had observed the first SLF in onchocercomas from hyperreactive patients.

Immune cells in secondary lymph follicles

The main cell types in the ectopic SLF were B lymphocytes (Fig. 9). Around and less so within the SLF, CD3-positive T cells were present (Figs. 5 and 6), which were CD45ROpositive in the germinal centre (Fig. 7). A subset of CD57positive T cells occurred in the germinal centre (Fig. 8) and some in the mantle zone. CD20-positive B cells predominated in the germinal centre and were also seen in the mantle zone (Fig. 9). We observed calprotectin-positive young macrophages in the infiltrate around the SLF but not in the germinal centre (Fig. 10). Mature CD68-positive macrophages were found dispersed in the germinal centre (Fig. 11) and several of them were tingible body macrophages phagocytosing degenerated cells (Fig. 12). Characteristic for SLF, CD35-positive FDCs formed a network in the germinal centre (Fig. 13). Mast cells positive for tryptase (Fig. 14) and IgE (not shown), eosinophils positive for eosinophilic cationic protein (Fig. 15), and neutrophils positive for defensin (Fig. 16) or cathepsin G (not shown) did not occur in the SLF or only in small numbers. However, these cells were frequently found in other zones of the onchocercomas, especially around blood vessels and dead $\mathrm{mf}$.
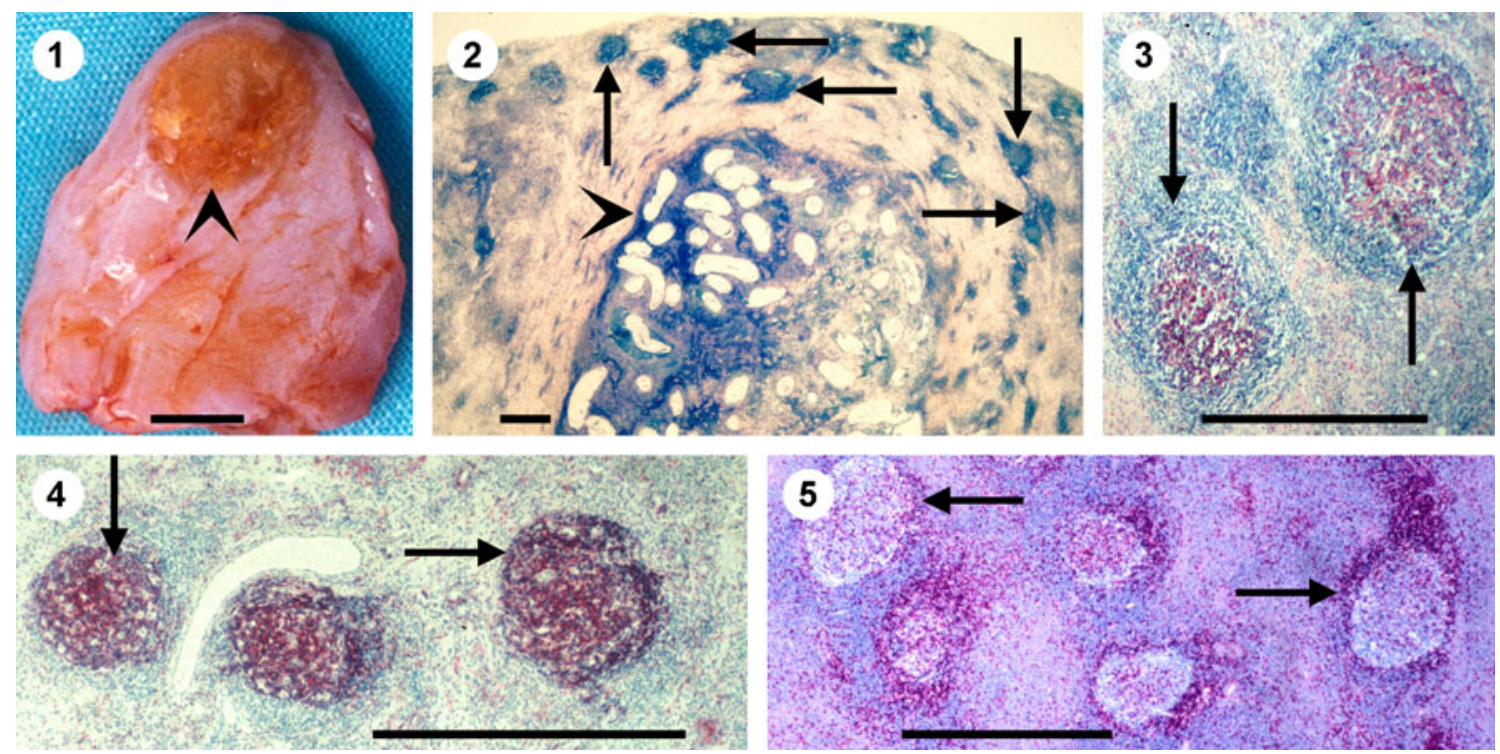

Figs. 1-5 Nodules with ectopic secondary lymph follicles from patients with hyperreactive onchocerciasis. 1 A typical hyperreactive onchocercoma from a 13-year-old girl with a small worm centre (arrowhead) surrounded by much nodule tissue. 2 A nodule from a 7-year-old girl showing much reactive tissue with many SLF (arrows)

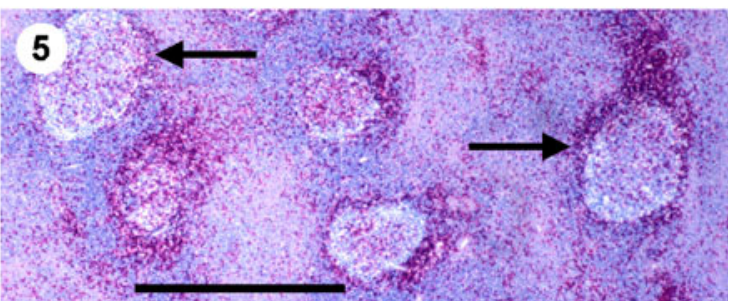

surrounding a small worm centre (arrowhead). Giemsa stain. 3-4 Consecutive sections from a nodule of a 9-year-old German boy with many SLF (arrows), in which IgE (3) and HLA-DR (4) are expressed in the germinal centre. 5 The same nodule as (2) with SLF showing CD3positive T lymphocytes in the mantle zone (arrows). Scale bar $1 \mathrm{~mm}$ 

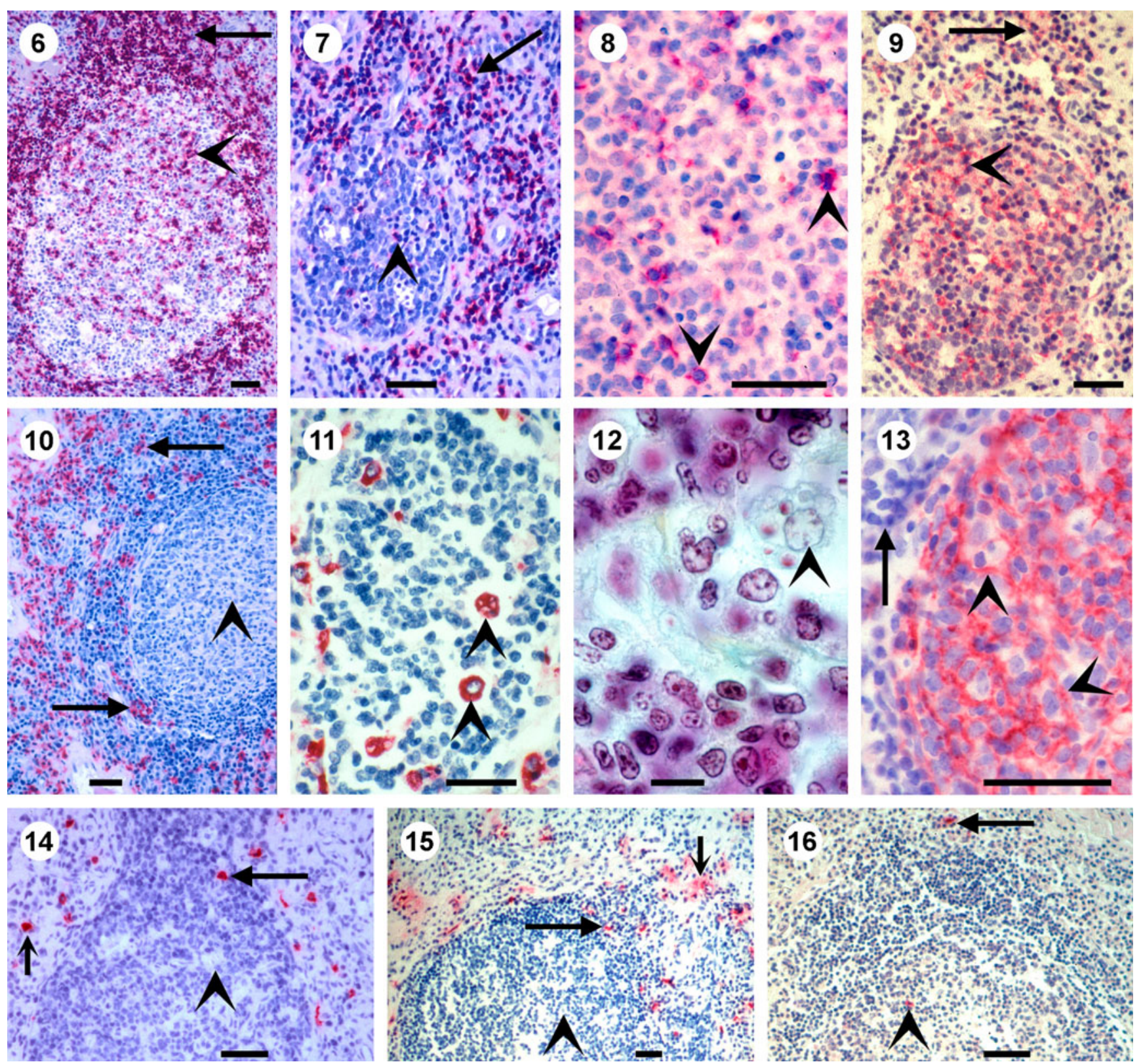

Figs. 6-16 Identification of different cell types in hyperreactive onchocercomas with secondary lymph follicles using immunohistology. 6-7 CD3-positive (6) and CD45Ro-positive (7) T cells mainly in the mantle zone (arrows) and few in the germinal centre (arrowheads). 7 From a 10-year-old girl. 8 CD57-positive T cells (arrowheads) in the germinal centre. $9 \mathrm{CD} 20$-positive B cells mainly in the germinal centre (arrowhead) and some in the mantle zone (arrow). 10 Calprotectin-positive young macrophages are seen around the SLF and in the mantle zone (arrows) whereas the germinal centre does not show these cells (arrowhead). 11 Mature CD68-positive macrophages in the germinal centre (arrowheads) in the nodule from a 9-year-old German boy. 12 Tingible body macrophages with phagocytosed leukocytes (arrowhead) in the germinal centre of the nodule from the boy. Movat stain. 13 CD35-positive FDCs form a network in the germinal centre (arrowheads) but are nearly absent in the mantle zone (arrow). 14-16 Tryptase-positive mast cells (14), eosinophil cationic protein-positive eosinophils (15) and few defensin-positive neutrophils (16) are seen in the mantle zone (arrows) but not in the germinal centre (arrowheads). Scale bar 40 and $10 \mu \mathrm{m}$ for (12)

in the SLF (Figs. 17 and 20). Also in other zones of the onchocercomas around blood vessels lymphocytes expressed TGF-beta, among them probably CD3-positive $\mathrm{T}$ cells (compare Figs. 18 and 19). In contrast to the weakly expressed TGF-beta, the antigen-presenting MHC class II

ly expressed by many lymphocytes and some macrophages 

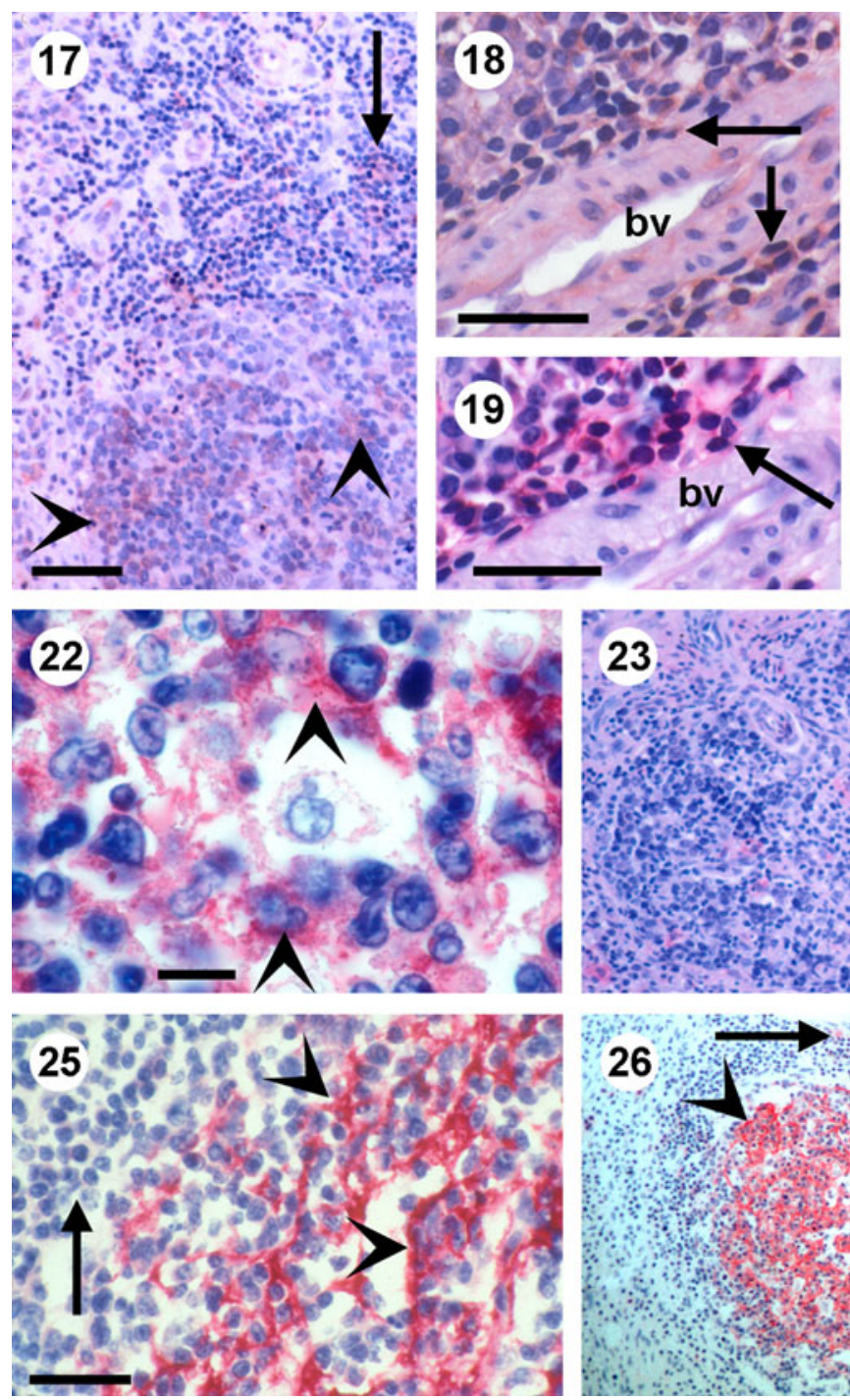
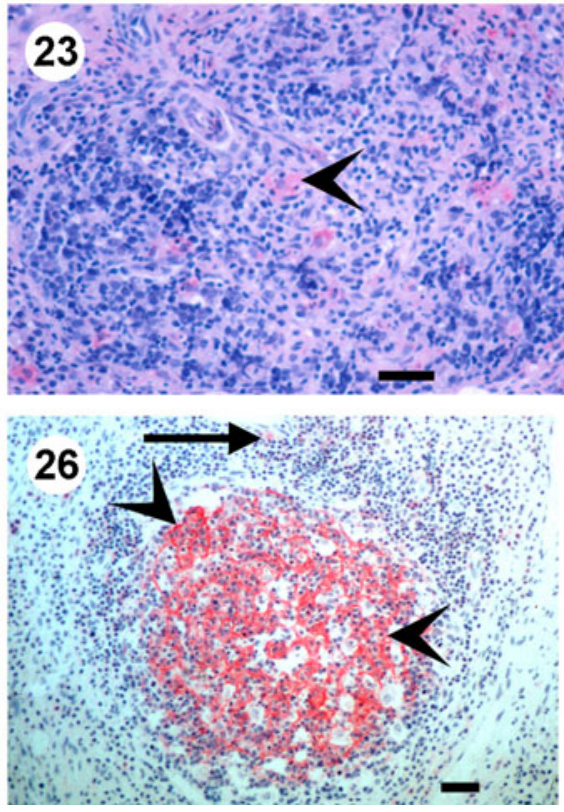
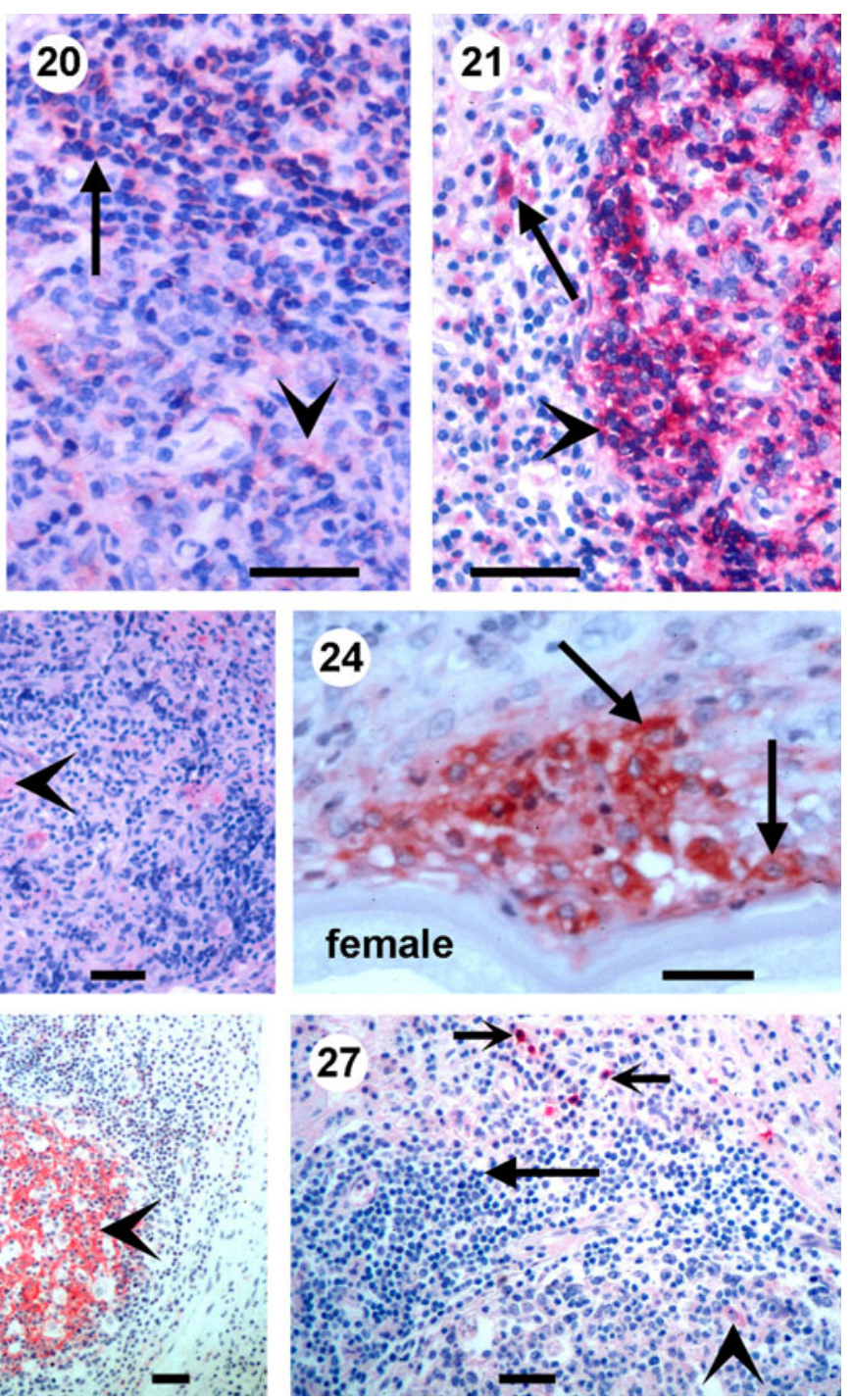

Figs. 17-27 Expression of TGF-beta, HLA-DR, alpha-1antichymotrypsin and immunoglobulins in hyperreactive onchocercomas with secondary lymph follicles. 17-19 TGF-beta is only weakly expressed by lymphocytes in the germinal centre (arrowheads in 17) and nearly not in the mantle zone (arrow) of an SLF, whereas lymphocytes around a blood vessel $(b v)$ are moderately TGF-betapositive (arrow in 18) in the same nodule of a 27-year-old woman; most of them are probably CD3-positive T cells as a consecutive section shows (arrow in 19). 20 Weakly TGF-beta-positive lymphocytes in the mantle zone (arrow) and the germinal centre (arrowhead) in the nodule of a 7-year-old girl. 21-22 Many HLA-DR-positive lymphocytes in the germinal centre (arrowheads) and in the mantle zone and few positive macrophages in the mantle zone (arrow in 21) in the nodule of a 7-year-old girl. 23-24 alpha1-antichymotrypsin is only weakly expressed in macrophages in the SLF (arrowhead in 23) but strongly near to a vital female filaria (arrows in 24). 25 A network of FDCs expressing $\operatorname{IgE}$ in the germinal centre (arrowheads) whereas the mantle zone is negative (arrow). 26 CD23-positive low-affinity IgE (epsilon) receptors are labelled in the germinal centre (arrowheads) in a section consecutive to (3). The mantle zone is negative (arrow). 27 Few IgG1positive plasma cells in the mantle zone (long arrow), the germinal centre (arrowhead) and more near to the edge of the SLF (short arrows) in the nodule of a German boy. Scale bar 40 and $10 \mu \mathrm{m}$ for (22) molecule HLA-DR, which is expressed by B cells, macrophages and FDCs, formed a strong network in the germinal centre (Figs. 21 and 22). The macrophages in the germinal centre only weakly expressed the acute-phase protein alpha1antichymotrypsin (Fig. 23), whereas most neutrophils and some macrophages adjacent to adult worms and to degenerated mf were strongly labelled (Fig. 24). Most lymphocytes and macrophages in the SLF did not express histamine
(Online Resource ESM1) but these cells were strongly histamine-positive adjacent to damaged $\mathrm{mf}$ (ESM2). Scattered plasma cells produced IgE in the mantle zone (not shown). In the germinal centre, IgE-positive FDCs formed a characteristic network (Figs. 3 and 25), which corresponded to the expression of $\mathrm{CD} 23$, the low-affinity $\operatorname{IgE}$ (epsilon) receptor (Fig. 26). Only few plasma cells produced $\mathrm{IgG1}$ (Fig. 27), IgG2 (ESM3) or IgG4 (ESM4) in the SLF. IgG1 
was more produced in other zones of the hyperreactive nodule, where it was attached to the cuticle of degenerated mf (ESM5). On the other hand, many plasma cells produced IgG4 (ESM6) in onchocercomas from hyporeactive patients. IgA expression by few plasma cells was observed in some lymphocyte infiltrations (not shown).

We know that microfilaricidal treatment has an influence not only on the clinical features but also on the occurrence of immune cells in the nodules. But our diethylcarbamazinetreated patients only came back after some months when new $\mathrm{mf}$ or new adult worms had appeared. We did not observe differences regarding the immune cells in the SLF between treated and untreated patients (not shown).

\section{Discussion}

Hyperreactive onchocerciasis is rare, probably less than $1 \%$ of the infected individuals, and we only detected SLF in seven of 63 (12\%) hyperreactive onchocercomas and none in hyporeactive nodules in Liberia. Can factors be identified which may contribute to the development of hyperreactivity and SLF formation? There is no indication that the filaria presents such a factor. For the hyperreactive patients in Yemen it was previously suggested that Onchocerca species other than $O$. volvulus might cause hyperreactivity. All filariae in our hyperreactive nodules, including those with SLF, from Yemen, Liberia and Ghana were morphologically $O$. volvulus, which we can differentiate from seven other Onchocerca species that we have examined by histology. DNA analysis of filariae from patients with hyperreactive onchocerciasis in Yemen, however, confirmed the diagnosis $O$. volvulus (Peter Fischer, Washington University, St. Louis, USA; personal communication). Furthermore, in the endemic area in Liberia so far no other Onchocerca species have been observed. Genetically different "strains" of $O$. volvulus occur usually in different geographic regions, whereas hyperreactive onchocerciasis occurs in Yemen, Sudan, Uganda, Liberia, Ghana, Benin, Guinea, Guatemala and probably other countries. Therefore host factors appear more probable. The dominance of the female gender in Liberia was obvious. The seven patients with SLF were all girls or women and 50 of $68(74 \%)$ Liberian and 31 of 39 (79\%) Ghanaian patients with hyperreactive onchocerciasis were female. But this may indicate rather cultural habits than biological causes since in Yemen, the men dominated with 43 of $54(80 \%)$ hyperreactive patients. So far the most probable differences observed concern genes associated with immunologically active components such as the HLA-DR molecules (Meyer et al. 1994) and a specific SNP Arg110Gln variant in the IL-13 gene (Hoerauf et al. 2002) known to lead to higher Th2 signalling, but other genetic factors associated with induction of the stronger Th2-dependent inflammation seen in sowda may still remain to be found. In contrast, hyporeactivity mainly depends on the high worm load.

The results from the eight examined nodules matched the histological findings described for onchocercomas from hyperreactive patients in Yemen and Liberia (e.g. Büttner and Racz 1983; Connor et al. 1983) and they were also in agreement with the rather few published immunohistological results (e.g. Brattig et al. 2009; Korten et al. 1998; Korten et al. 2010a). Exceptions were the SLF in these onchocercomas, as we found these structures only in the regional lymph nodes of onchocerciasis patients (Knab et al. 1997; Racz et al. 1983). The SLF are a characteristic component of the lymph nodes presenting features typical for the different forms of onchocerciasis.

Regional and especially femoral lymph nodes from hyperreactive patients are enlarged with diameters of $5 \mathrm{~cm}$ and more. They show extensive hyperplasia of the follicles (Abdel-Hameed et al. 1987; Connor et al. 1983; Racz et al. 1983) and the germinal centres present a network of IgEtrapping FDCs. No or only few $\mathrm{mf}$ are seen in the lymph nodes from untreated hyperreactive patients because most of the $\mathrm{mf}$ have already been eliminated in onchocercomas or skin. In contrast, patients with longstanding hyporeactive infections increasingly develop fibrotic lymph nodes with finally only few small SLF (Gibson and Connor 1978; Knab et al. 1997; Racz et al 1983). The SLF in regional lymph nodes from patients with intermediate forms are neither reduced by fibrosis nor do they show an extensive hyperplasia.

The immune cell populations in the ectopic SLF equalled those in onchocerciasis lymph nodes and often also those in other ectopic locations. The young calprotectin-positive macrophages occurred outside of the SLF and in the outer mantle zone. Calprotectin is an endogenous Toll-like receptor 4 agonist, playing a role during amplification of inflammation (Ehrchen et al. 2009). In contrast, the CD68positive mature macrophages were present in the germinal centre and some of them were tingible body macrophages indicating apoptosis and subsequent phagocytosis of activated cells. Such activated cells may be centrocytes (B cells) in the germinal centres, which interact with antigen held by FDCs and undergo high death rates in the SLF (MacLennan 1994). Mast cells were also present in the mantle zone of the SLF as in onchocercal lymphadenitis (Racz et al. 1983). Otherwise mast cells are rarely described in ectopic SLF, e.g. in rheumatoid arthritis (Timmer et al. 2007). Since they were located outside the germinal centres in the T-cell-rich zones of the SLF and primarily influence the Th1/2 axis, we assume a Th2-driving, IgE-mediated function here as already stated for hyperreactive onchocercomas (Korten et al. 1998). This is in line with the occurrence of eosinophils around and in the mantle zone and in other zones of onchocercomas with 
mf-producing worms (Wildenburg et al. 1996) or dead filariae. Neutrophils were nearly absent in the SLF but they were abundant near older female worms, in the pseudocysts with male filariae and attached to dead $\mathrm{mf}$ in accordance with previous studies (Korten et al. 1998). The neutrophils are attracted by compounds from the Wolbachia endobacteria harboured by the filariae (Brattig et al. 2001), which do not occur in the SLF.

The predominance of B lymphocytes in the SLF corresponds to the strong antibody responses documented for the hyperreactive onchocerciasis (Brattig 2004; Brattig et al. 1994; Büttner et al. 1982; Gallin et al. 1995; Mpagi et al. 2000). The characteristic IgE network of FDCs expressing the low-affinity $\operatorname{IgE}$ (epsilon) receptor correlated well to the IgE presence in onchocercomas, lymph nodes and peripheral blood and represents a key feature of hyperreactive onchocerciasis (Racz et al. 1983; Timmann et al. 2003). The IgE antibodies mainly refer to polyclonal $\operatorname{IgE}$ and in addition to Onchocerca protein-specific IgE (Mpagi et al. 2000). No intracytoplasmatic expression of IgG subclasses was observed in the SLF although two subclasses are present in high concentrations in sera from hyperreactive patients (Brattig et al. 1994). They are produced in other zones of the onchocercomas and in lymph nodes, more IgG1 and IgG3 by hyperreactive patients and more IgG4 by hyporeactive patients with high $\mathrm{mf}$ loads. It is known that IgG4 is preferably induced by regulatory $\mathrm{T}$ cell responses (Satoguina et al., 2008).

The formation of ectopic SLF may contribute to the pathogenesis of hyperreactive onchocerciasis, e.g. the strong humoral response (Brattig 2004; Büttner et al. 1982) and the extended cellular infiltrates characteristic for hyperreactive onchocercomas, skin and lymph nodes (Büttner and Racz 1983; Connor et al. 1983; Racz et al. 1983). An increase of cellular infiltration is also reported for rheumatoid arthritis (Timmer et al. 2007). One potential role of ectopic SLF is to expand inflammation or defence locally, often associated with an exacerbated state of autoimmune disease (López De Padilla et al. 2009).

SLF increase the probability that antigens encounter specific lymphocyte subsets. If the immune system is not able to create an efficient response against local antigens, it is an advantage to produce an SLF at the site of infection. The factors contributing to the generation of ectopic SLF induce a chronic stimulation of the immune system involving antibody-producing cells. There are other similarities between SLF in onchocercomas from hyperreactive patients reported here and the observations in other diseases. The B cell attracting chemokine-1 (CXCL13), a ligand of CXCR-5, is a major inductor for the formation of germinal centres in the synovium of chronic arthritis (Shi et al. 2001) leading to high antibody production including autoantibody formation, which is also characteristic for hyperreactive onchocerciasis as autoantibodies against defensin show (Gallin et al. 1995).

The expression of immunosuppressive TGF-beta, the proinflammatory tumour necrosis factor-alpha (TNF-alpha) and compounds such as alphal-antichymotrypsin, histamine and HLA-DR varied in the ectopic SLF in onchocercomas and it differed from that in lymph nodes from hyporeactive patients (Knab et al. 1997; Korten et al. 2010a). Since TGF-beta suppresses other immune cells, also in helminth infections (Ince et al. 2009), its low expression in the SLF here corresponds with the hyperreactive status as in other onchocercal tissues in skin and lymph nodes of hyperreactive patients in contrast to strong TGF-beta expression in these tissues from hyporeactive patients (Brattig et al. 2009; Korten et al. 2010a, b).

Alpha1-antichymotrypsin was produced in macrophages adjacent to the $\mathrm{mf}$ and adult worms but not in the SLF of the examined onchocercomas. This is in accordance with observations in lymph nodes from untreated and ivermectin-treated onchocerciasis patients (Knab et al. 1997). The enzyme is a serine proteinase inhibitor, which can cleave cathepsin $G$ that we observed in the neutrophils adjacent to adult worms and damaged mf. Thus it contributes to the protection of host tissues from damaging enzymes released by other host cells. Furthermore, the cells in the SLF did not produce histamine, which triggers local inflammatory reactions, and which we observed in the macrophages and lymphocytes near damaged $\mathrm{mf}$ as also previously reported for these cells and mast cells in nodules from hyperreactive patients (Korten et al. 1998).

In contrast to the previous compounds, the HLA-DR complex was strongly expressed by B cells in the germinal centre and in macrophages and B cells adjacent to dead $\mathrm{mf}$. Furthermore, lymphocytes and macrophages in and adjacent to the mantle zone and macrophages, giant cells and neutrophils near to a living productive female $O$. volvulus strongly expressed tumour necrosis factor-alpha in nodule tissues from our untreated patients 6 and 3 in Table 1 (ESM7-8; Hoerauf and Büttner, unpublished). TNF-alpha is a cytokine involved in systemic inflammation and participates in immune responses in SLF and the damaged neutrophils adjacent to the female worm may be related to the induction of cell death by TNF-alpha (Locksley et al. 2001).

In conclusion, the ectopic SLF in onchocercomas from hyperreactive patients are equally composed as those in lymph nodes from onchocerciasis patients and they resemble SLF in other infectious and autoimmune diseases. The extensive expression of HLA-DR and IgE in conjunction with cytotoxic and phagocytosed cells in the germinal centre and Th2-directed cells in the mantle zone suggests that these ectopic SLF contribute to the strong local amplification of the Th2-biased immunopathology in the 
hyperreactive form of onchocerciasis, which is both, an infectious and an autoimmune disease. The weak expression of the immunosuppressive TGF-beta is in accordance with the hyperreactivity.

Acknowledgements We thank Ingeborg Albrecht, Frank Geisinger and Gudrun Großschupff for technical assistance. We are obliged to Prof. Kassa Darge (Dept for Pediatric Radiology, University Würzburg, Germany), who treated the German boy and sent us the nodule. We are obliged to the physicians and laboratory assistants, who participated in the care for the patients in Liberia. Prof. Paul Racz (BNI) previously discussed the observations with us and commented on the manuscript.

The Ethics Commission of the Medical Board, Hamburg, had approved nodulectomies for research purposes. Nodulectomies were also generally supported by the World Health Organisation (World Health, 1987). The procedures used were in accordance with the Declaration of Helsinki (1975 and its revision in 1983).

\section{References}

Abdel-Hameed AA, Noah MS, Schacher JF, Taher SA (1987) Lymphadenitis in sowda. Trop Geogr Med 39:73-76

Albiez EJ, Gallin M, Erttmann KD, Racz P, Büttner DW (1985) Characteristics of chronic severe onchodermatitis in Liberia. J Liberian Med Assoc 15:120-125

Albiez EJ, Büttner DW, Duke BOL (1988) Diagnosis and extirpation of nodules in human onchocerciasis. Trop Med Parasitol 39:331346

Brattig NW (2004) Pathogenesis and host responses in human onchocerciasis: impact of Onchocerca filariae and Wolbachia endobacteria. Microbes Infect 6:113-128

Brattig NW, Krawietz I, Abakar AZ, Erttmann KD, Kruppa TF, Massougbodji A (1994) Strong IgG isotypic antibody response in sowdah type onchocerciasis. J Infect Dis 170:955-961

Brattig NW, Büttner DW, Hoerauf A (2001) Neutrophil accumulation around Onchocerca worms and chemotaxis of neutrophils are dependent on Wolbachia endobacteria. Microbes Infect 3:439446

Brattig NW, Schwohl A, Hoerauf A, Büttner DW (2009) Identification of the lipid mediator prostaglandin $E_{2}$ in tissue immune cells of humans infected with the filaria Onchocerca volvulus. Acta Trop 112:231-235

Büttner DW, Racz P (1983) Macro- and microfilariae in nodules from onchocerciasis patients in the Yemen Arab Republic. Tropenmed Parasitol 34:113-121

Büttner DW, von Laer G, Mannweiler E, Büttner M (1982) Clinical, parasitological and serological studies on onchocerciasis in the Yemen Arab Republic. Tropenmed Parasitol 33:201-212

Büttner DW, Albiez EJ, von Essen J, Erichsen J (1988) Histological examination of adult Onchocerca volvulus and comparison with the collagenase technique. Trop Med Parasitol 39:390-417

Cerroni L, Borroni RG, Massone C, Chott A, Kerl H (2007) Cutaneous B-cell pseudolymphoma at the site of vaccination. Am J Dermatopathol 29:538-542

Colli C, Leinweber B, Müllegger R, Chott A, Karl H, Cerroni L (2004) Borrelia burgdorferi-associated lymphocytoma cutis: clinicopathologic, immunophenotypic, and molecular study of 106 cases. J Cutan Pathol 31:232-240

Connor DH, Gibson DW, Neafie RC, Merighi B, Buck AA (1983) Sowda-onchocerciasis in north Yemen: a clinicopathologic study of 18 patients. Am J Trop Med Hyg 32:123-137

Darge K, Büttner DW (1995) Ivermectin treatment of hyperreactive onchodermatitis (sowda) in Liberia. Trop Med Parasitol 46:206-212
Ehrchen JM, Sunderkötter C, Foell D, Vogl T, Roth J (2009) The endogenous Toll-like receptor 4 agonist S100A8/S100A9 (calprotectin) as innate amplifier of infection, autoimmunity, and cancer. J Leukoc Biol 86:557-566

Erttmann KD (1987) Long-time treatment of onchocerciasis patients with severe skin lesions using diethylcarbamacine citratrate. (In German) Dissertation, University Hamburg

Gallin MY, Jacobi AB, Büttner DW, Schönberger O, Marti T, Erttmann KD (1995) Human autoantibody to defensin: disease association with hyperreactive onchocerciasis (sowda). J Exp Med 182:41-47

Gibson DW, Connor DH (1978) Onchocercal lymphadenitis: clinicopathologic study of 34 patients. Trans Roy Soc Trop Med Hyg $72: 137-154$

Hoerauf A, Brattig N (2002) Resistance and susceptibility in human onchocerciasis-beyond Th1 vs. Th2. Trends Parasitol 18:2531

Hoerauf A, Kruse S, Brattig NW, Heinzmann A, Mueller-Myhsok B, Deichmann KA (2002) The variant Arg 110GLn of human IL-13 is associated with an immunologically hyper-reactive form of onchocerciasis (sowda). Microbes Infect 4:37-42

Ince MN, Elliott DE, Setiawan T, Metwali A, Blum A, Chen HL, Urban JF, Flavell RA, Weinstock JV (2009) Role of T cell TGFbeta signalling in intestinal cytokine responses and helminthic immune modulation. Eur J Immunol 39:1870-1878

Knab J, Darge K, Büttner DW (1997) Immunohistological studies on macrophages in lymph nodes of onchocerciasis patients after treatment with ivermectin. Trop Med Int Health 2:1156-1169

Korten S, Wildenburg G, Darge K, Büttner DW (1998) Mast cells in onchocercomas from patients with hyperreactive onchocerciasis (sowda). Acta Trop 70:217-231

Korten S, Hoerauf A, Kaifi JT, Büttner DW (2010a). Low levels of transforming growth factor-beta (TGF-beta) and reduced suppression of Th2-mediated inflammation in hyperreactive human onchocerciasis. Parasitology, in press

Korten S, Kaifi JT, Büttner DW, Hoerauf A (2010b) Transforming growth factor-beta expression by host cells is elicited locally by the filarial nematode Onchocerca volvulus in hyporeactive patients independently from Wolbachia. Microbes Infect. doi:10.1016/j.micif.2010.03.011 (Epub ahead of print)

Locksley RM, Killeen N, Lenardo MJ (2001) The TNF and TNF receptor superfamilies: integrating mammalian biology. Cell 104:487-501

López de Padilla CM, Vallejo AN, Lacomis D, McNallan K, Reed AM (2009) Extranodal lymphoid microstructures in inflamed muscle and disease severity of new-onset juvenile dermatomyositis. Arthritis Rheum 60:1160-1172

MacLennan IC (1994) Germinal centers. Annu Rev Immunol 12:117_ 139

Maubec E, Pinquier L, Vigier M, Caux F, Amsler E, Aractingi S, Chafi H, Janin A, Cayuela JM, Dubertret L, Authier FJ, Bachelez H (2005) Vaccination-induced cutaneous pseudolymphoma. J Am Acad Dermatol 52:623-629

Mazzucchelli L, Blaser A, Kappeler A, Schärli P, Laissue JA, Baggiolini M, Uguccioni M (1999) BCA-1 is highly expressed in Helicobacter pylori-induced mucosa-associated lymphoid tissue and gastric lymphoma. J Clin Invest 104:R49-R54

Meyer CG, Gallin M, Erttmann KD, Brattig N, Schnittger L, Gelhaus A, Tannich E, Begovich AB, Erlich HA, Horstmann RD (1994) HLA$\mathrm{D}$ alleles associated with generalized disease, localized disease, and putative immunity in Onchocerca volvulus infection. Proc Natl Acad Sci U S A 91:7515-7519

Mpagi JL, Büttner DW, Tischendorf FW, Erttmann KD, Brattig NW (2000) Humoral responses to a secretory Onchocerca volvulus protein: differences in the pattern of antibody isotypes to recombinant Ov20/OvS1 in generalized and hyperreactive onchocerciasis. Parasite Immunol 22:455-460 
Racz P, Tenner-Racz K, Luther B, Büttner DW, Albiez EJ (1983) Immunopathologic aspects in human onchocercal lymphadenitis. Bull Soc Pathol Exot Fil 6:676-680

Ratnatunga N, de Witesundera MS (1999) Histopathological diagnosis of subcutaneous Dirofilaria repens infection in humans. Southeast Asian J Trop Med Public Health 30:375-378

Rubio de Krömer MT, Medina-De la Garza CE, Brattig NW (1995) Differences in eosinophil and neutrophil chemotactic responses in sowda and generalized form of onchocerciasis. Acta Trop 60:21-33

Satoguina JS, Adjobimey T, Arndts K, Hoch J, Oldenburg J, Layland LE, Hoerauf A (2008) Tr1 and naturally occurring regulatory T cells induce IgG4 in B cells through GITR/GITR-L interaction, IL-10 and TGF-beta. Eur J Immunol 38:3101-3113

Shi K, Hayashida K, Kaneko M, Hashimoto J, Tomita T, Lipsky PE, Yoshikawa H, Ochi T (2001) Lymphoid chemokine B cellattracting chemokine-1 (CXCL13) is expressed in germinal center of ectopic lymphoid follicles within the synovium of chronic arthritis patients. J Immunol 166:650-655
Specht S, Brattig N, Büttner M, Büttner DW (2009) Criteria for the differentiation between young and old Onchocerca volvulus filariae. Parasitol Res 105:151-158

Timmann C, Abraha RS, Hamelmann C, Buttner DW, Lepping B, Marfo Y, Brattig N, Horstmann RD (2003) Cutaneous pathology in onchocerciasis associated with pronounced systemic T-helper 2-type responses to Onchocerca volvulus. Br J Dermatol 149:782-787

Timmer TC, Baltus B, Vondenhoff M, Huizinga TW, Tak PP, Verweij CL, Mebius RE, van der Pouw Kraan TC (2007) Inflammation and ectopic lymphoid structures in rheumatoid arthritis synovial tissues dissected by genomics technology: identification of the interleukin-7 signaling pathway in tissues with lymphoid neogenesis. Arthritis Rheum 56:2492-2502

Wildenburg G, Krömer M, Büttner DW (1996) Dependence of eosinophil granulocytes infiltration into nodules on the presence of microfilariae producing Onchocerca volvulus. Parasitol Res $82: 117-124$

World Health Organization (1987). Expert committee on onchocerciasis. WHO Tech Rep Ser 752, WHO, Geneva 